\title{
Comportamento Balístico de Compósito de Polietileno de Altíssimo Peso Molecular: Efeito da Radiação Gama
}

\author{
Andreia L. S. Alves, Lucio F. C. Nascimento, João Carlos Miguez Suarez \\ Departamento de Engenharia Mecânica e de Materiais, IME
}

\begin{abstract}
Resumo: Os materiais compósitos de matriz polimérica (PMCs) reforçados por fibras são considerados excelentes materiais de engenharia. Em aplicações estruturais, quando uma elevada relação resistência peso é fundamental para o projeto, os PMCs vêm substituindo com sucesso diversos materiais convencionais. Materiais têxteis são utilizados, desde a $2^{\text {a }}$ Guerra Mundial, como blindagens balísticas. Materiais fabricados com fibra do polietileno de altíssimo peso molecular (UHMWPE) são empregados na produção de blindagens, para proteção pessoal e em carros de combate. Todavia, em virtude de terem sido desenvolvidos e comercializados mais recentemente, não existem informações suficientes sobre o desempenho balístico desses materiais após a sua exposição aos agentes ambientais. No presente trabalho foi estudado o comportamento balístico de placas compósitas fabricadas com fibra de polietileno de altíssimo peso molecular (UHMWPE), após sua exposição à radiação gama. Os resultados dos testes balísticos foram relacionados com as alterações macromoleculares induzidas pela irradiação por meio de ensaios mecânicos (dureza, impacto e flexão) e físico-químicos (espectroscopia no infravermelho, calorimetria diferencial de varredura e análise termogravimétrica). Foi verificado que a irradiação gama provoca modificações nas cadeias macromoleculares do polímero, que alteram as propriedades mecânicas do compósito de UHMWPE, reduzindo, nas doses de radiação mais elevadas, o seu desempenho balístico. Estes resultados são apresentados e discutidos.
\end{abstract}

Palavras-chave: Comportamento balístico, polietileno de altíssimo peso molecular, UHMWPE, radiação gama, degradação.

\section{Ballistic Behaviour of Ultra-High Molecular Weight Polyethylene: Effect of Gamma Radiation}

Abstract: The fiber reinforced polymer matrix composites (PMCs) are considered excellent engineering materials. In structural applications, when a high strength-to-weight ratio is fundamental for the design, PMCs are successfully replacing many conventional materials. Since World War II textile materials have been used as ballistic armor. Materials manufactured with ultrahigh molecular weight polyethylene (UHMWPE) fibers are used in the production of armor materials, for personnel protection and armored vehicles. As these have been developed and commercialized more recently, there is not enough information about the action of the ionizing radiation in the ballistic performance of this armor material. In the present work the ballistic behavior of composite plates manufactured with ultrahigh molecular weight polyethylene (UHMWPE) fibers were evaluated after exposure to gamma radiation. The ballistic tests results were related to the macromolecular modifications induced by the environmental degradation through mechanical (hardness, impact and flexure) and physicochemical (infrared spectroscopy, differential scanning calorimetry and thermal gravimetric analysis) tests. Our results indicate that gamma irradiation induces modifications in the UHMWPE macromolecular chains, altering the mechanical properties of the composite and decreasing, for higher radiation doses, its ballistic performance. These results are presented and discussed.

Keywords: Ballistic behavior, ultrahigh molecular weight polyethylene, UHMWPE, gamma radiation, degradation.

\section{Introdução}

Desde os primórdios da história, o ser humano busca criar e aperfeiçoar mecanismos que o protejam das agressões dos inimigos ${ }^{[1]}$. A possibilidade de se formular o material adequado para cada aplicação foi possível desde o advento dos materiais compósitos, que têm encontrado emprego em diversos segmentos industriais, tais como aeronáutica, aeroespacial, de transportes, de material bélico, de material esportivo etc. Fibras e tecidos têm sido usados mundialmente, a partir da década de 1970, como materiais de reforço em compósitos e os materiais compósitos de matriz polimérica (PMCs) reforçados com fibras são, atualmente, considerados excelentes materiais de engenharia ${ }^{[2,3]}$.

Materiais poliméricos, tais como poliéster, poliamida, aramida, polietileno e polipropileno, vem sendo largamente utilizados na industria de material bélico. Tecidos poliméricos são empregados, desde a $2^{\mathrm{a}}$ Guerra Mundial, como blindagens balísticas, quer sob a forma de blindagens flexíveis (soft armor), quer sob a forma de blindagens rígidas (hard armor). A tendência atual nos projetos de blindagens está direcionada para uma melhor relação

Autor para correspondência: João Carlos Miguez Suarez, Instituto Militar de Engenharia, Praça General Tibúrcio 80, CEP: $22290-270$, Rio de Janeiro, RJ. E-mail:jmiguez@taurus.ime.eb.br 
proteção/peso ${ }^{[4]}$, e, em conseqüência, os PMCs por possuírem uma maior resistência específica, vêm substituindo, com sucesso, diversos materiais de blindagem tradicionais. A blindagem rígida ou blindagem têxtil laminada, formada por camadas de tecido unidas por uma resina, comporta-se, no impacto balístico, como um material conjugado, pois além de resistirem parcialmente à ação de penetração do projétil, absorvem a energia de impacto resultante do choque do projétil com o alvo ${ }^{[5-8]}$. Presentemente, blindagens compósitas fabricadas com fibra de polietileno de altíssimo peso molecular (ultra-high molecular weight polyethyleneUHMWPE) são empregadas, face à sua baixa densidade e elevada tenacidade, em proteções pessoais (coletes e capacetes) e em blindagens de veículos de uso militar e civil ${ }^{[9-11]}$.

É sabido que os materiais poliméricos, quando expostos às radiações ionizantes, apresentam modificações estruturais, que produzem, comumente, redução nas suas propriedades tecnológicas ${ }^{[12,13]}$. O desempenho balístico de blindagens de UHMWPE, após a sua exposição à essas radiações, não está perfeitamente conhecido, face ao seu desenvolvimento e fabricação mais recentes. Em conseqüência torna-se necessária a realização de estudos visando desenvolver técnicas de caracterização e de análise do comportamento destes materiais ao longo da sua vida útil.

No presente trabalho o comportamento balístico de placas compósitas de polietileno de altíssimo peso molecular (UHMWPE), após exposição à radiação gama, foi analisado com o auxílio de ensaios mecânicos (dureza, impacto e flexão) e físico-químicos (espectroscopia no infravermelho, calorimetria de varredura diferencial e análise termogravimétrica).

\section{Experimental}

\section{Materiais}

Foi estudado um material compósito, projetado e produzido pela empresa Stopower Sistemas de Segurança Ltda (Curitiba, PR), para emprego como blindagem Classe II ${ }^{[14,15]}$. A blindagem compósita, na forma de placas quadradas de $270 \mathrm{~mm}$ de lado e com $5 \mathrm{~mm}$ de espessura, foi fabricada com 30 camadas de Spectra Shield ${ }^{\mathbb{R}}$ LCR, unidas unidirecionalmente por moldagem a quente, na pressão de $8 \mathrm{~kg} / \mathrm{cm}^{2}$ e na temperatura de $120^{\circ} \mathrm{C} \sim 125^{\circ} \mathrm{C}$. O Spectra Shield ${ }^{\mathbb{R}} \mathrm{LCR}$, tecido não-tecido (non-woven fabric) produzido pela empresa Honeywell Inc. (Petersburg, VA, USA), é formado por duas lâminas unidirecionais de fibras de polietileno de altíssimo peso molecular (UHMWPE) com cadeia estendida, tipo Spectra ${ }^{\circledR}$, dispostas cruzadas, uma em relação à outra, na orientação $0^{\circ} / 90^{\circ}$, intercaladas com uma película de poliuretano e revestidas por uma resina termoplástica (Figura 1). As características comerciais ${ }^{[16]}$ e as determinadas experimentalmente ${ }^{[17]}$ para o Spectra Shield ${ }^{\mathbb{B}}$ LCR empregado na blindagem compósita estão apresentadas na Tabela 1.

\section{Irradiação}

O material foi irradiado ao ar em um equipamento dotado

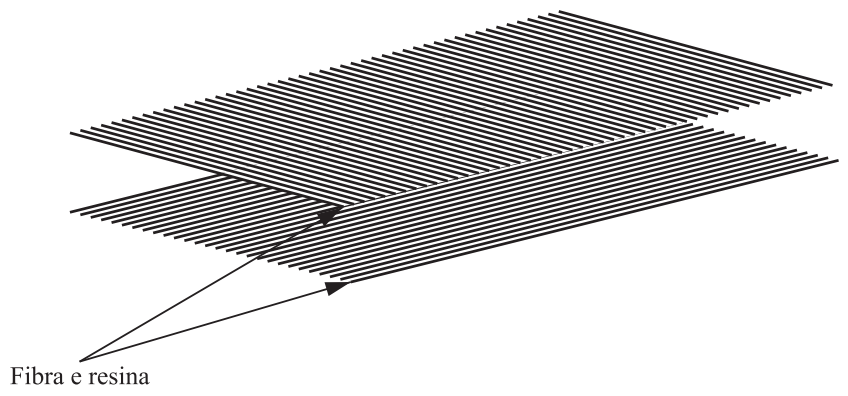

Figura 1. Desenho esquemático do Spectra Shield ${ }^{\circledR}$

Tabela 1. Características do tecido Spectra Shield ${ }^{\circledR}$ LCR

\begin{tabular}{lcccc}
\hline \multirow{2}{*}{ Características } & \multicolumn{2}{c}{ Dados comerciais ${ }^{[16]}$} & \multicolumn{2}{c}{ Dados experimentais ${ }^{[17]}$} \\
\cline { 2 - 5 } & Método & Valor & Método & Valor \\
\hline \multirow{2}{*}{ Espessura (mm) } & ASTM & \multirow{2}{*}{$0,18 \pm 0,05$} & ISO & 0,23 \\
& D1777/64 & & NBR & \\
Gramatura (g/m $\left.{ }^{2}\right)$ & ASTM & \multirow{2}{*}{$150 \pm 10$} & $10591 / 98$ & 153,83 \\
& D5034/95 & & ASTM & 17,88 \\
Resistência à tração (kgf) & - & - & D5034/95 \\
& - & - & ASTM & 2,74 \\
Alongamento (\%) & - & - & D5034/95. \\
\hline
\end{tabular}

de uma fonte de ${ }^{137} \mathrm{Cs}$, em uma taxa de dose de 2,0 kGy/h, tendo recebido as doses de $25 \mathrm{kGy}$ e $250 \mathrm{kGy}$. Estas doses foram selecionadas considerando a transição dúctil-frágil que ocorre no UHMWPE, conforme mostrado pela variação da sua plasticidade ${ }^{[18]}$.

\section{Ensaios mecânicos}

O ensaio de dureza Rockwell foi realizado em um durômetro marca Pantec modelo RBS segundo a norma ASTM D785 ${ }^{[19]}$, utilizando-se a escala $\mathrm{R}$ (60 $\mathrm{kg}$ e esfera de aço de $12,7 \mathrm{~mm}$ ). Foram realizadas 5 impressões em cada placa.

O ensaio de impacto foi realizado conforme a norma ASTM D5942 ${ }^{[20]}$, na temperatura ambiente, em uma máquina pendular de ensaio, marca Panambra, modelo PW 30/15R, sistema Wolpert-Amsler, com capacidade máxima de 300 J. Dois grupos, um transversal ao outro, de corpos de prova tipo Charpy, de $80 \mathrm{~mm} \times 12 \mathrm{~mm} \times 5 \mathrm{~mm}$, tendo um entalhe central de $45^{\circ}$ e $4 \mathrm{~mm}$ de altura, em posição normal às camadas do compósito, e um rasgo de $1 \mathrm{~mm}$ de profundidade na face oposta ao entalhe, foram produzidos, por corte mecânico, a partir das placas. Foram testados, para cada condição e sentido, 5 corpos de prova. Foi determinada uma energia específica (energia por unidade de área), considerada igual a energia absorvida no impacto do corpo de prova dividida pela sua área resistente. Este procedimento foi realizado visando a obtenção de um valor comparativo para o comportamento sob impacto, pois, devido à elevada tenacidade do compósito de UHMWPE, o rompimento dos corpos de prova Charpy pode não ocorrer.

O ensaio de flexão em 3 pontos, segundo a norma ASTM

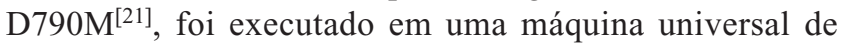
ensaios marca Emic, modelo DL2000, à temperatura de $21^{\circ} \mathrm{C}$ 
Tabela 2. Resultados obtidos nos ensaios mecânicos do compósito (valor médio), antes e após irradiação

\begin{tabular}{|c|c|c|c|c|}
\hline Condição & Dureza Rockwell R & $\begin{array}{l}\text { Energia específica } \\
\qquad\left(\mathbf{J} / \mathbf{m}^{2}\right)\end{array}$ & $\begin{array}{c}\text { Tensão para } 5 \% \\
\text { de deformação } \\
\text { (MPa) }\end{array}$ & $\begin{array}{c}\text { Módulo de } \\
\text { elasticidade } \\
\text { (MPa) }\end{array}$ \\
\hline Como recebido & 43 & $1418^{(1)}$ & 9,6 & 866,8 \\
\hline Irradiado com $25 \mathrm{kGy}$ & 29 & $2180^{(1)}$ & 8,8 & 833,8 \\
\hline Irradiado com $250 \mathrm{kGy}$ & 87 & $1745^{(2)}$ & 23,6 & 2907,2 \\
\hline
\end{tabular}

Observações:

(1) Não ocorreu a fratura dos corpos de prova Charpy;

(2) Ocorreu a fratura dos corpos de prova Charpy.

e na velocidade de ensaio de $1,30 \mathrm{~mm} / \mathrm{s}$. Para cada condição, foram retirados, por corte, dois grupos de corpos de prova por placa, um transversal ao outro, nas dimensões de $110 \mathrm{~mm} \mathrm{x}$ $10 \mathrm{~mm} \times 5 \mathrm{~mm}$. Foram testados, para cada condição e sentido, 7 corpos de prova, determinando-se a tensão máxima para $5 \%$ de deformação e o módulo de elasticidade.

\section{Ensaios físico-químicos}

A espectroscopia no infravermelho (FTIR) foi executada em um espectrômetro Perkin-Elmer, modelo 1720x, na região entre $4000 \mathrm{~cm}^{-1}-400 \mathrm{~cm}^{-1}$, empregando-se a técnica de reflectância interna múltipla (MIR) com um cristal KRS-5 a $45^{\circ}$ e um detetor de TGS. O espectro foi obtido com resolução de $2 \mathrm{~cm}^{-1}, 40$ varreduras em cada ensaio e processado em um gerenciador de dados Perkin-Elmer (IRDM). As amostras para o ensaio do polímero irradiado foram extraídas das faces frontal e posterior de cada placa, enquanto que no material "como recebido" as amostras foram retiradas apenas da face frontal.

A calorimetria diferencial de varredura (DSC) foi realizada conforme o prescrito na norma ASTM D3417 ${ }^{[22]}$, empregando-se um equipamento Shimatzu, modelo DSC-50, calibrado com índio $\left(\mathrm{T}_{\mathrm{m}}=156,6^{\circ} \mathrm{C}\right.$ e $\left.\Delta \mathrm{H}_{\mathrm{f}}=6,8 \mathrm{cal} / \mathrm{g}\right)$, tendo um sistema computarizado de análise. Foram retiradas amostras da face frontal de cada grupo de placas, com peso entre $5 \mathrm{mg}$ e $8 \mathrm{mg}$, que foram submetidas, na velocidade de $20^{\circ} \mathrm{C} / \mathrm{min}$, a um duplo ciclo de aquecimento/resfriamento de $30^{\circ} \mathrm{C}$ até $250{ }^{\circ} \mathrm{C}$, em uma atmosfera de nitrogênio. O grau de cristalinidade foi calculado utilizando o calor de fusão do polietileno perfeitamente cristalino igual a $289,3 \mathrm{~J} / \mathrm{g}^{[23]}$.

A análise termogravimétrica (TGA) foi realizada em um equipamento Shimadzu modelo TGA-50, dispondo de um par termoelétrico de cromel-alumel. A calibração do termopar foi realizada com alumel $\left(\mathrm{T}_{\mathrm{m}}=163{ }^{\circ} \mathrm{C}\right)$, níquel $\left(\mathrm{T}_{\mathrm{m}}=354{ }^{\circ} \mathrm{C}\right)$ e perkalloy $\left(\mathrm{T}_{\mathrm{m}}=596^{\circ} \mathrm{C}\right)$. As amostras retiradas da face frontal de cada grupo de placas, pesando entre $5 \mathrm{mg}$ e $8 \mathrm{mg}$, foram colocadas em cadinhos de platina e aquecidas, sob nitrogênio, de $30{ }^{\circ} \mathrm{C}$ até $650{ }^{\circ} \mathrm{C}$, na velocidade de $20^{\circ} \mathrm{C} / \mathrm{min}$.

\section{Ensaio balístico}

O ensaio balístico, antes e após exposição das placas à radiação gama, foi executado com munição de $9 \mathrm{~mm}$ FMJ, projétil de 8,0g, de acordo com as normas NEB/T E-316 ${ }^{[14]}$ e NIJ Std $0108.01^{[15]}$. No teste balístico foi ensaiada uma placa por con- dição, que, apoiada em um bloco de argila especial, "plastilina", foi submetida a 3 impactos.

\section{Resultados e Discussão}

\section{Ensaios mecânicos}

Os resultados dos ensaios mecânicos tiveram pequena variação em relação ao sentido de retirada dos corpos de prova, longitudinal e transversal, mostrando que o processo de produção empregado na fabricação das placas permite a obtenção de um material praticamente ortotrópico. Os valores médios das propriedades determinadas nos ensaios mecânicos, para cada condição das placas e calculados independentemente do sentido de extração dos corpos de prova, estão apresentados na Tabela 2. Os resultados mostram que a exposição à radiação gama influencia o comportamento mecânico do compósito de UHMWPE.

A resistência ao impacto do compósito variou com a dose de radiação gama; os corpos de prova do material "Como recebido" e "Irradiado com $25 \mathrm{kGy}$ " não romperam no ensaio de impacto, enquanto que ocorreu a fratura nos "Irradiado com 250kGy". Verifica-se que as placas compósitas irradiadas com $25 \mathrm{kGy}$ tem maior tenacidade, medida pela energia específica de deformação, que as "Como recebido". A energia específica, na condição "Irradiado com 250kGy", foi calculada considerando-se a energia de ruptura dos corpos de prova, não podendo ser comparada com a das demais condições, onde a energia específica foi determinada em função da energia de deformação dos corpos de prova. Todavia pode-se afirmar, conforme mostrado pelo rompimento dos corpos de prova Charpy, que o material "Irradiado com 250kGy" é menos tenaz, ou seja mais frágil, que o "Como recebido" e o "Irradiado com 25kGy".

O comportamento sob impacto, a resistência à flexão e o módulo de elasticidade variam, para as mesmas condições, com a dureza; quanto menor a dureza, maior a energia absorvida no impacto, maior a tenacidade e menores a resistência mecânica e o módulo de elasticidade. Verifica-se que as propriedades mecânicas mostraram tendências similares e coerentes, indicando que o comportamento mecânico do compósito de UHMWPE é influenciado de uma maneira uniforme pelas alterações moleculares induzidas no polímero pela exposição à radiação gama.

\section{Ensaios físico-químicos}

Os parâmetros determinados nos ensaios físico-químicos 
(FTIR, DSC e TGA), índice de oxidação (IO), temperatura de fusão $\left(\mathrm{T}_{\mathrm{m}}\right)$, grau de cristalinidade $\left(\mathrm{X}_{\mathrm{c}}\right)$ e temperatura de início da variação de massa (onset) $\left(\mathrm{T}_{\mathrm{i}}\right)$, estão mostrados na Tabela 3.

A Figura 2 mostra os espectros no infravermelho do UHMWPE, antes e após a irradiação. A comparação dos espectros permite verificar que, para vibrações características do polietileno, grupo metilênico $\left(\mathrm{CH}_{2}, 1463 \mathrm{~cm}^{-1}\right)$ e deformação angular no plano $\left(720 \mathrm{~cm}^{-1}\right)$, a transmitância varia em função da exposição à radiação gama. Esta variação pode ser relacionada à cisão das cadeias macromoleculares, que é iniciada com o rompimento das ligações $\mathrm{C}-\mathrm{H}$. O espectro de infravermelho mostra, ainda, a existência de uma banda de absorção em $1712 \mathrm{~cm}^{-1}$, resultante da formação de grupos carbonila oriundos da degradação oxidativa das cadeias ${ }^{[24,25]}$. O índice de oxidação, uma relação entre as absorções das bandas de $1712 \mathrm{~cm}^{-1}$ e $1463 \mathrm{~cm}^{-1[26,27]}$ e que está apresentado na Tabela 3, mostra que a degradação oxidativa no compósito varia com a dose de radiação gama; na irradiação com $25 \mathrm{kGy}$ ocorre, predominantemente, na face frontal, enquanto que, para a dose de $250 \mathrm{kGy}$, acontece ao longo de toda a espessura da placa. Estes resultados são coerentes considerando que a degradação oxidativa causada pela irradiação gama é controlada por um processo de difusão, donde a profundidade de penetração aumenta com a dose de radiação ${ }^{[28,29]}$.

Os parâmetros térmicos, temperatura de fusão $\left(\mathrm{T}_{\mathrm{m}}\right)$ e grau de cristalinidade $\left(\mathrm{X}_{\mathrm{c}}\right)$, determinados por calorimetria diferencial de varredura (DSC), e a temperatura de início da va-
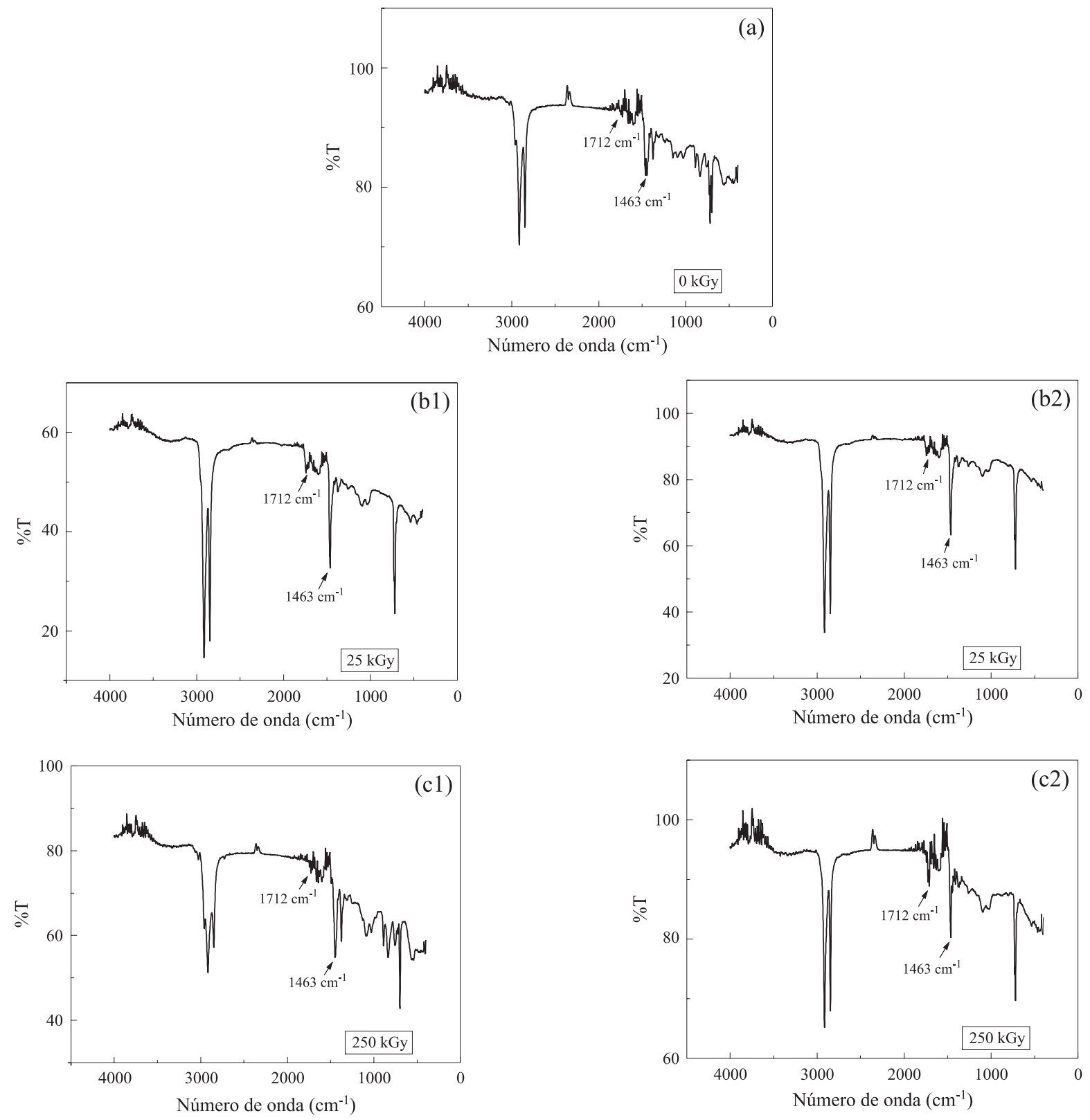

Figura 2. Espectros do infravermelho (FTIR-MIR) do UHMWPE, antes e após irradiação (a) "Como recebido"; (b) Irradiado com 25kGy; (c) Irradiado com 250kGy; (1) face frontal; (2) face posterior 
Tabela 3. Índice de oxidação $(\mathrm{IO})$, temperatura de fusão $\left(\mathrm{T}_{\mathrm{m}}\right)$, grau de cristalinidade $\left(\mathrm{X}_{\mathrm{c}}\right)$ e temperatura de início da variação de massa $\left(\mathrm{T}_{\mathrm{i}}\right)$ do $\mathrm{UHMWPE}$, antes e após irradiação

\begin{tabular}{lccccc}
\hline \multicolumn{1}{c}{ Condição } & Face & IO & $\mathbf{T}_{\mathrm{m}}\left({ }^{\circ} \mathbf{C}\right)$ & $\mathbf{X}_{\mathbf{c}}(\boldsymbol{\%})$ & $\mathbf{T i}\left({ }^{\circ} \mathbf{C}\right)$ \\
\hline Como recebido & Frontal & 0,36 & 140,0 & 23 & 438,5 \\
\hline \multirow{2}{*}{ Irradiado com 25kGy } & Frontal & 0,67 & 135,6 & 35 & 454,1 \\
& Posterior & 0,29 & - & - & 45 \\
\hline \multirow{2}{*}{ Irradiado com 250kGy } & Frontal & 0,66 & 131,2 & - & 455,8 \\
& Posterior & 0,51 & - & - \\
\hline
\end{tabular}

riação de massa (onset) $\left(\mathrm{T}_{\mathrm{i}}\right)$, determinada por análise termogravimétrica (TGA), estão mostrados, também, na Tabela 3. A redução da temperatura de fusão com a irradiação pode ser atribuída à cisão e à oxidação das cadeias macromoleculares, o que modifica a posição e o formato das curvas endotérmicas. A cristalinidade cresce após exposição à radiação; a cisão de cadeias macromoleculares produz cadeias mais curtas e de menor peso molecular, que, face terem uma maior capacidade de empacotamento, se recristalizam com maior facilidade aumentando a cristalinidade do UHMWPE ${ }^{[30-32]}$. Um aumento na cristalinidade induz, usualmente, uma redução na tenacidade do polímero, tornando-o mais frágil. Todavia, as placas irradiadas com $25 \mathrm{kGy}$, apesar de exibirem um aumento na cristalinidade, possuem um comportamento mais tenaz do que o material "Como recebido", conforme mostrado pelos resultados do ensaio de impacto Charpy. Este comportamento indica que os mecanismos de cisão e reticulação das cadeias macromolecures, que ocorrem com a exposição do UHMWPE à radiação gama em baixas doses de radiação, tem maior influencia na tenacidade do que o aumento da cristalinidade. A trinca principal, ao encontrar as interfaces das regiões cristalinas surgidas com a redução no tamanho das cadeias, é dividida em trincas secundárias que se pro- pagam em várias direções. A divisão da trinca principal em segmentos menores causa um retardo na velocidade de propagação da mesma, aumentando a energia específica do compósito $^{[33,34]}$. A redução da tenacidade com o aumento da dose de radiação, de $25 \mathrm{kGy}$ para $250 \mathrm{kGy}$, mostra, conforme indicado pelo aumento do índice de oxidação, que os mecanismos de degradação oxidativa tem, nas doses maiores, maior influência sobre a tenacidade do que a cisão e a reticulação.

Observa-se, ainda, que a irradiação produz um aumento na temperatura de início da variação de massa, indicando um aumento na estabilidade térmica do UHMWPE. À medida que acontece a cisão das cadeias com a irradiação, que tornam-se mais curtas e mais semelhantes, ocorre a formação de um material mais ordenado com interações mais rígidas e, em conseqüência, mais estável, o que melhora o comportamento dinâmico do polímero ${ }^{[32,35,36]}$, confirmando o observado no ensaio de impacto e a discussão dos resultados do DSC.

\section{Ensaio balístico}

A avaliação do desempenho de uma blindagem depende da interação do projétil com o alvo, e compreende, basicamente, a verificação da ocorrência ou não da sua falha e a determinação dos danos produzidos pelo impacto balístico.

Tabela 4. Resultados do ensaio balístico (munição de 9mm FMJ e projétil de 8,0g), antes e após irradiação

\begin{tabular}{lccccc}
\hline \multirow{2}{*}{ Condição } & Tiro & $\begin{array}{c}\text { Velocidade do projétil } \\
(\mathbf{m} / \mathbf{s})\end{array}$ & Desempenho & \multicolumn{2}{c}{ Área de danos $\left(\mathbf{c m}^{2}\right)$} \\
\cline { 5 - 6 } & 1 & 427,89 & Não perfurou & 5,92 & Média \\
\hline \multirow{2}{*}{ Como recebido } & 2 & 379,69 & Não perfurou & 7,20 & 6,25 \\
& 3 & 394,78 & Não perfurou & 5,60 & \\
\hline Irradiado com 25kGy & 1 & 369,64 & Perfurou & 9,36 & \\
& 2 & 369,45 & Não perfurou & 12,06 & \\
& 3 & 373,25 & Perfurou & 6,65 & \\
\hline & 4 & 375,05 & Não perfurou & 6,39 & 40,77 \\
\hline
\end{tabular}



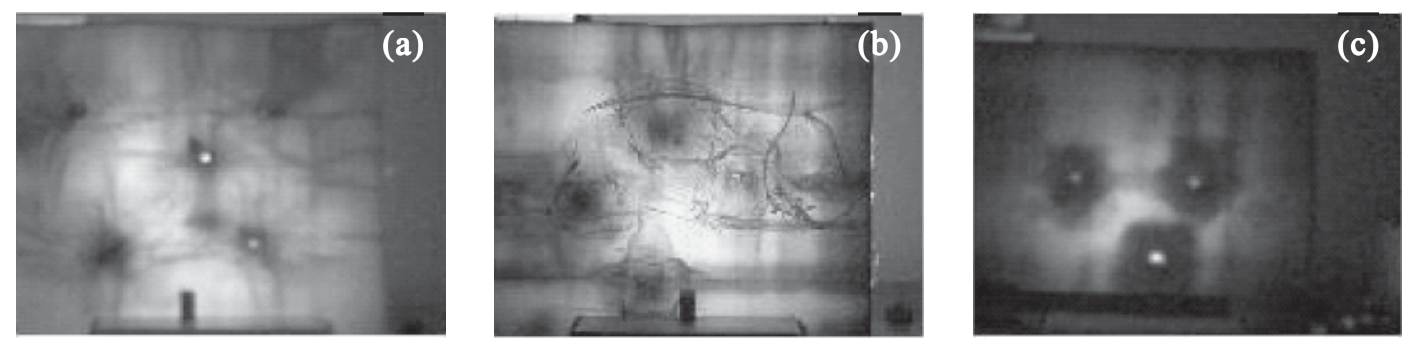

Figura 3. Fotografias das placas submetidas ao ensaio balístico (face posterior): (a) "Como recebida"; (b) Irradiada com 25kGy; (c) Irradiada com 250kGy

A falha de uma blindagem é analisada, normalmente, em função da ocorrência ou não de penetração. A penetração, termo utilizado para se definir a entrada de um projétil em um alvo, pode ser parcial ou total. Na penetração parcial o projétil atinge o alvo sem atravessá-lo, enquanto que na penetração total ou completa ocorre a sua perfuração. A avaliação do que significa a ocorrência ou não de uma perfuração depende do critério de avaliação empregado. Existem 3 critérios para se avaliar a falha de uma blindagem: do Exército, da Marinha e o de Proteção, cada um definindo, de um modo particular, o significado de uma perfuração (penetração completa) ${ }^{[37]}$. Neste trabalho foi utilizado o "Critério da Marinha", que estabelece "uma perfuração ocorre quando o projétil ou a maior parte dele passa completamente pela blindagem".

A avaliação dos danos produzidos na blindagem pelo impacto do projétil inclui a determinação dos mecanismos de deformação e a medição do tamanho da área danificada.

Os resultados do ensaio balístico com a munição de $9 \mathrm{~mm}$ FMJ estão apresentados na Tabela 4, onde verifica-se que as placas "Como recebido" não apresentaram perfuração, enquanto que as irradiadas foram perfuradas, pelo menos parcialmente.

O exame visual das placas, realizado após o ensaio balístico, revelou que o compósito, mesmo quando não perfurado, sofreu danos que aumentaram com a irradiação. O principal mecanismo de deformação foi a delaminação, que ocorreu nas placas "Como recebido" e "Irradiada com 25kGy". Foi verificado que na placa irradiada a delaminação aconteceu, predominantemente, nas camadas frontais, evidenciando uma maior degradação superficial resultante, provavelmente, da degradação oxidativa. Na perfuração a falha da placa irradiada com $25 \mathrm{kGy}$ ocorreu com uma separação fibra-matriz, enquanto que, na placa irradiada com $250 \mathrm{kGy}$, a falha foi devido ao trincamento da matriz.

Para uma blindagem translúcida, como é o caso das placas compósitas deste trabalho, a medição do tamanho da área do dano pode ser realizada por meio de um método fotográfico $^{[38]}$. A colocação de uma fonte de luz na parte posterior da placa submetida ao ensaio balístico produz, na região danificada que surge em torno do ponto de impacto do projétil (área delaminada), uma imagem mais escura, cuja área pode ser medida na cópia fotográfica. A Figura 3 apresenta as fotografias da face posterior de cada uma das placas submetidas ao ensaio balístico, onde é possível identificar a área de danos produzida por cada impacto balístico. Foi realizada a medição de cada uma dessas áreas, calculando-se, por placa, um valor médio para a área de danos (Tabela 4). Para esta determinação as fotografias receberam um tratamento da imagem realizado com o auxílio do programa MicroStation $\mathrm{SE}^{\mathbb{B}[39]}$, que melhorou o contraste da mancha de delaminação. Verifica-se que a área de danos aumenta com a irradiação, crescendo, sensivelmente, para a placa irradiada com a dose de $250 \mathrm{kGy}$.

\section{Conclusões}

Os resultados do trabalho mostraram que a metodologia utilizada é adequada para avaliar o efeito da irradiação gama no comportamento balístico de blindagens fabricadas com compósitos de matriz polimérica reforçada com fibras de polietileno de altíssimo peso molecular (UHMWPE).

Tendo em vista a análise realizada pode-se, ainda, apresentar as seguintes conclusões:

1. A irradiação gama do compósito de UHMWPE produz, devido à degradação oxidativa do polímero, diferenças significativas nas propriedades mecânicas; reduzindo, principalmente, a sua tenacidade.

2. No compósito de UHMWPE o principal mecanismo de absorção da energia de impacto é a delaminação das camadas, independentemente da condição do material.

3. O aumento da dose de radiação altera o modo de falha do compósito de UHMWPE, de separação fibra-matriz para trincamento da matriz.

4. A diminuição na capacidade de absorção de impactos do compósito de UHMWPE, após sua exposição a doses mais elevadas de radiação gama, reduz o desempenho balístico da blindagem.

5. As placas fabricadas com 30 camadas de Spectra Shield e expostas à radiação gama, em doses mais elevadas, são perfuradas por munição $9 \mathrm{~mm}$ FMC, não sendo, neste caso, adequadas para emprego como blindagem Tipo II segundo a norma NIJ Std 0108.01.

6. A exposição à radiação gama influencia o comportamento balístico do compósito de UHMWPE, devendo, em conseqüência, ser executado um controle periódico deste tipo de blindagem para verificar se a degradação resultante não vai comprometer a sua capacidade de proteção.

\section{Agradecimentos}

Os autores agradecem às agências financiadoras FAPERJ, CNPq e CAPES e, pelo fornecimento das placas, à Stopower Sistemas de Segurança Ltda. 


\section{Referências Bibliográficas}

1. ST 17-1-3, "History and role of armor", US Army Armor School, Fort Knox, Kentucky, p.1, (1974).

2. Mano, E.B. - "Polímeros como materiais de engenharia", Ed. Edgard Blücher Ltda., São Paulo, SP, p. 124, (1991).

3. Chawla, K.K. - "Composite materials: science and engineering”, Springer-Verlag, Inc., New York, NY, $2^{\text {nd }}$ Ed., p.1, (1998).

4. Chin, A.S.C. - Mater. Sci. Engng, A259, p.155 (1999).

5. Roberts, J. - "The use of fibre reinforced composite materials in land systems equipment", in: Proc. of Defence Components and Equipment Exhibition, Session 3 - New Materials, p.6.1, London, (1985).

6. Miguez Suarez, J. C. - Revista Militar de Ciência e Tecnologia, 3 (4), p.46, (1986).

7. Savage, G.M. - Metals and Materials, 5 (May), p.285, (1989).

8. Egglestone, G.T.; Gellert, E.P. \& Woodward, R.L. 'Perforation failure mechanisms in laminated composites", in: Proc. of Conference on Materials United in the Service of Man, Vol. 1, p.2.1, Perth, Australia, (1990).

9. Desper, C.R.; Cohen, S.H. \& Cohen, A.O. - J. Appl. Polym. Sci., 47, p.1129, (1993).

10. Lee, B.L.; Song, J.W. \& Ward, J.E. - J. Comp. Mater., 28, p.1202, (1994).

11. Gellert, E.P.; Pattie, S.D. \& Woodward, R.L - J. Mater. Sci., 33, p.1845, (1998).

12. Billlmeyer Jr., F.W. - "Textbook of Polymer Science”, John Wiley \& Sons, Inc., New York, NY, p.141, (1984).

13. Grassie, N. \& Scott, G. - "Polymer Degradation and Stabilization", Cambridge University Press, Cambridge, (1985).

14. Norma NEB/T E-316 - "Proteção Balística de Carros de Passeio" - Exército Brasileiro, Rio de Janeiro, RJ, (2001).

15. Norma NIJ Std 0108.01 - "Ballistic Resistant Protective Materials" - National Institute of Justice, U. S. Department of Justice - Washington, DC, (1985).

16. Catalogo comercial da Honeywell Inc.

17. Relatório de Ensaio n ${ }^{\circ} 772 / 02$ - Centro de Tecnologia da Indústria Química e Têxtil - CETIQT / SENAI / CNI, Rio de Janeiro, RJ, (2002).

18. Miguez Suarez, J. C. \& de Biasi, R. S. - Polym. Deg. Stab., 82, p.221, (2003).

19. Norma ASTM D785 - Rockwell Hardness of Plastics and Electrical Insulating Materials - Standard Method of Test for - American Society for Testing and Materials, Philadelphia, PA, (1993).

20. Norma ASTM D5942 - Determining Charpy Impact Strength of Plastics - Standard Method of Test for-American Society for Testing and Materials, Philadelphia, PA, (1993).
21. Norma ASTM D790M - Flexural Properties of Unreinforced and Reinforced Plastics and Electrical Insulating Materials [Metric] - Standard Test Methods for - American Society for Testing and Materials, Philadelphia, PA, (1993).

22. Norma ASTM D3417 - Heat Fusion and Crystallization of Polymers by Thermal Analysis - Standard Test for - American Society for Testing and Materials, Philadelphia, PA, (1983).

23. Wunderlich, B. \& Cormier, C.M. - J. Polym. Sci.: Part A-2, 5, p.987, (1967).

24. DeVries, K.L.; Smith R.H. \& Fanconi, B.M. - Polymer, 21, p.949, (1980).

25. Miguez Suarez, J.C.; Monteiro, E.E.C. \& Mano, E.B. - Polym. Deg. Stab., 75 , p.143, (2002).

26. Kurtz, S.M; Muratoglu, O.K.; Evans, M. \& Edidin, A.A. Biomaterials, 20, p.1659, (1999).

27. Premnath, A.; Bellare, V.; Merril, E.W.; Jasty, M. \& Harris, W.H. - Polymer, 40, p.2215, (1999).

28. Spadaro, G. - Eur. Polym. J., 29, p.851, (1993).

29. Miguez Suarez, J.C. \& Mano, E.B. - Polym. Deg. Stab., 72 , p.217, (2001).

30. Klein, P.G.; Gonzalez-Orozco, J.A. \& Ward, I.M. - Polymer, 32, p.1732, (1991).

31. Zhao, Y.; Luo, Y. \& Jiang, B. - J. Appl. Polym. Sci., 50, p.1797, (1993).

32. Miguez Suarez, J.C., Mano, E.B. \& Pereira, R.A. - Polym. Deg. Stab., 69, p.217, (2000).

33. Cook, J. \& Gordon, J.E. - Proc. Royal Soc., A282, p.508, (1964).

34. Miguez Suarez, J.C. \& Mano, E.B. - "Influência da radiação gama na tenacidade de misturas de polietilenos reciclados", in: Anais do $57^{\circ}$ Congresso Anual da ABM - Internacional (CDROM), p.1069, São Paulo, SP, jul (2002).

35. Goldman, M.; Gronsky, R.; Ranganathan R. \& Pruitt, L. Polymer, 37, p.2909, (1996).

36. Miguez Suarez, J.C.; Mano, E.B. \& Tavares, M.I.B. - J. Appl. Polym. Sci., 78, p.899, (2000).

37. Backman, M.E. \& Goldsmith, W. - Int. J. Engng. Sci., 16, p.265, (1978).

38. Alves, A.L.S.; Nascimento, L.F.C. \& Miguez Suarez, J.C. 'Comportamento mecânico e balístico de blindagem compósita de matriz polimérica", in: Anais do $58^{\circ}$ Congresso Anual da ABM (CDROM), p.1758, Rio de Janeiro, RJ, jul (2003).

39. MicroStation SE Program ${ }^{\circledR}$, Bentley Systems, Inc., Exton, PA, USA, (1995).

Enviado: $28 / 10 / 03$

Reenviado: $23 / 03 / 03$

Aprovado: 26/03/03 